\title{
Diez años de incidentes críticos pediátricos declarados en el Sistema Español de Notificación en Seguridad en Anestesia y Reanimación
}

\author{
Analysis of the pediatric critical incidents declared in ten \\ years time in the Spanish Anesthesiology and Reanimation \\ Safety Notification System
}

Andrea Romera'. MD PhD, Alejandro Garrido. MD PhD.

\begin{abstract}
Objetives: The aim of this study is to analyze all the pediatric critical incidents (PCI) reported in the Spanish Anesthesiology and Reanimation Safety Notification System in ten years time. Materials and Methods: We reviewed all the critical incidents $(\mathrm{Cl})$ reported between the years 2009 and 2018 in SENSAR online database and we selected those related to newborns and children up to 16 years. Results: $9480 \mathrm{CI}$ were declared; 474 were pediatrics. Infants accounted for the $36 \%$ of the $\mathrm{PCl}$, of whom the $44 \%$ were children under the age of one. Most of them affected to healthy patients (the 66\%) and took place in the operating room (the $49 \%$ ), during general pediatric surgery (the $19 \%$ ), otorhinolaryngology (the 15\%), orthopedic (the 13\%) and radiology procedures (the $5 \%$ ). The majority of them were medications errors (the $24 \%$ ), followed by clinical incidents (the 18\%) and equipment-related events (14\%). Human factor accounted for the $42 \%$ of reports - both of them in its individual characteristics (the 25\%) and in team performance (the 15\%). Up to the 30\% of the children suffered consequences derived from the incidents. Two fatal events were recorded, both airway related. Improvement measures were designed and implemented in the $93 \%$ of them. Conclusions: Designing studies that shed light on the causes of the relative inefficiency of $\mathrm{CI}$ Reporting Systems and the factors that condition a lower rate of $\mathrm{Cl}$ communication in the pediatric setting in comparison with the adult population is necessary.
\end{abstract}

Key words:

Incident, pediatric, patient safety

Hospital General Universitario Gregorio Marañón de Madrid, Servicio de Anestesiología y Reanimación. España.

Fecha de recepción: 05 de abril de 2020

Fecha de aceptación: 14 de junio de 2020

ORCID

https://orcid.org/0000-0002-9062-2377

Correspondencia:

Dra. Andrea Romera

andreilla111@hotmail.com 


\section{RESUMEN}

Objetivos: Realizar un análisis retrospectivo de todos los incidentes críticos pediátricos (ICP) declarados en el Sistema Español de Notificación en Seguridad en Anestesia y Reanimación en sus primeros diez años. Material y Métodos: Se realizó una revisión de los incidentes críticos (IC) declarados entre el 1 de enero de 2009 y el 31 de diciembre de 2018. Se seleccionaron los IC acontecidos en pacientes de 0 a 16 años de edad. Resultados: De los 9.480 IC declarados en ese período, 474 fueron ICP. El 36\% ocurrió en lactantes menores de dos años, de los que el $44 \%$ fueron menores de un año. La mayoría (66\%) ocurrieron en pacientes sanos y tuvieron lugar en quirófano (49\%), durante procedimientos de cirugía general pediátrica (19\%), de otorrinolaringología (15\%), de traumatología (13\%) y de radiología (5\%). El tipo de IC más declarado fueron los errores de medicación (24\%), seguidos de los incidentes clínicos (18\%) y de los errores de equipamiento (14\%). El factor humano estuvo implicado en el $42 \%$ de los IC, tanto en su faceta del individuo (25\%) como en las relaciones en equipo (15\%). El 30\% de los pacientes sufrieron daño. Se registraron dos fallecimientos derivados del incidente, ambos relacionados con la vía aérea. Se implementaron medidas de mejora en un $93 \%$ de los casos. Conclusiones: Es necesario profundizar en las causas de la ineficiencia relativa de los Sistemas de Declaración de IC y en las que condicionan una menor tasa de comunicación de IC en el ámbito pediátrico respecto al adulto.

\section{Palabras clave:}

Incidente, pediatría, seguridad del paciente

\section{Introducción}

os Sistemas de Registro de Incidentes Críticos (SRIC) son una herramienta diseñada para obtener información de seguridad del paciente (SP) y transformarla en aprendizaje[1]. Aunque fueron introducidos en el ámbito sanitario en 1987[2], hasta 2009 no se creó el Sistema Español de Notificación en Seguridad en Anestesia y Reanimación (SENSAR). Se trata del primer sistema multihospital de comunicación, análisis y gestión de incidentes críticos (IC) en el ámbito de la anestesiología española, que en los últimos años se ha expandido también de forma internacional. El programa de comunicación de incidentes de SENSAR consta de una base de datos online constituida por notificaciones anónimas, voluntarias y confidenciales de IC declarados por anestesiólogos de 114 hospitales, 102 de los cuales están ubicados en España, 7 en Chile y 5 en Uruguay. Los incidentes notificados son estudiados por equipos de expertos locales mediante un análisis centrado en el sistema[3] que supera la cultura tradicional de la culpabilización del individuo y aboga por la detección de los fallos del sistema que pueden ser subsanados para evitar la repetición del incidente.

Los pacientes pediátricos tienen más riesgo de sufrir un IC que los pacientes adultos[4]. La literatura existente sobre incidentes críticos pediátricos (ICP) es escasa[5],[6], lo que ha llevado a varios autores a destacar la necesidad de realizar análisis de IC exclusivamente pediátricos e incluso de desarrollar SRIC específicamente pediátricos - enfocados en áreas de mejora de la anestesiología pediátrica que normalmente quedan diluidos en muestras más grandes y adultas - y a señalar que la toma de decisiones es más ágil en grupos de análisis pequeños y focalizados[7]. El objetivo de este estudio es realizar un análisis retrospectivo de todos los ICP declarados en SENSAR en sus primeros diez años de recorrido, partiendo de la base de datos general.

\section{Material y Métodos}

Se realizó una revisión de todos los IC declarados entre el 1 de enero de 2009 y el 31 de diciembre de 2018 en la base online de SENSAR. Se definió incidente crítico como cualquier evento que redujera o pudiera haber reducido el margen de seguridad del paciente[8]. Se seleccionaron todos los IC acontecidos en pacientes de 0 a 16 años de edad y se recogieron los siguientes parámetros epidemiológicos: edad, ASA, lugar donde ocurrió el IC, factores contribuyentes, tipo de cirugía, daño al paciente y posibilidad de implantar medidas de mejora. 
El sistema online de notificación de SENSAR se denomina PITELO. Todos los anestesiólogos de los hospitales acreditados por SENSAR pueden acceder a él desde cualquier ordenador, utilizando una clave específica de cada hospital. La comunicación de un IC comienza con una breve redacción de lo ocurrido y con el registro de los datos epidemiológicos básicos - edad, sexo, relación entre el comunicante y el IC -. A continuación se desglosan los posibles factores contribuyentes, tal y como los describe la clasificación de Vincent[9]:

1) Acción insegura inmediata:

- No acción insegura inmediata.

- Distracción: falta de atención.

- Distracción: por olvido.

- Equivocación: en aplicación de prácticas seguras.

- Equivocación: relacionada con el conocimiento.

- Transgresión de protocolo.

- Sabotaje.

2) Factores contribuyentes del paciente:

- Patología.

- Lenguaje y comunicación.

- Personalidad y factores sociales.

3) Factores contribuyentes del individuo:

- Conocimientos y habilidades.

- Competencia o cualificación.

- Salud física y mental.

- Prisa.

4) Factores contribuyentes de la tarea:

- Diseño.

- Protocolos.

- Pruebas.

- Ayuda en la toma de decisiones.

5) Factores contribuyentes del equipo humano:

- Comunicación verbal.

- Comunicación escrita.

- Supervisión y búsqueda de ayuda.

- Estructura.

6) Factores contribuyentes del lugar:

- Dotación de personal de distintas categorías.

- Turnos, carga de trabajo.

- Equipamientos, material, medicación.

- Problemas administrativos o de gestión.

- Ambiente.

- Físico.

7) Factores contribuyentes de organización y gestión - Financiación.
- Estructura organizativa.

- Políticas, normas u objetivos.

- Fallo en cultura de seguridad y prioridades.

8) Factores contribuyentes contexto institucional:

- Contexto económico y regulatorio o normativo.

- Sistema nacional de salud o consejería de Sanidad.

- Organismos reguladores externos.

Por último, el comunicante describe el daño que sufrió el paciente, estima la posibilidad de que ese mismo IC se repita y sugiere medidas de mejora. Aunque la declaración es anónima, el sistema ofrece la posibilidad de proporcionar un mail de contacto para obtener un feedback más estrecho.

Una vez comunicado un IC, todos los analizadores pertenecientes al hospital del declarante reciben una alerta por correo electrónico. De forma conjunta, generalmente en forma de reunión presencial, ese IC es puesto en común y analizado. Se diseñan medidas de mejora específicas e individualizadas y, una vez implantadas, se cierra el IC.

\section{Resultados}

9.480 IC fueron declarados en SENSAR entre el 2009 y el 2018, de los cuales 474 acontecieron en pacientes pediátricos, lo que supone el $5 \%$ del total.

En lo que respecta a la edad, el 36\% (175) ocurrió en lactantes menores de dos años; 78 de ellos eran menores de un año. La mayoría ocurrieron en pacientes sanos: el 45\% (213) en pacientes ASA-I, el $21 \%$ (100) en ASA-II, el 17\% (81) en ASA-III y el 4\% (19) en ASA-IV. Tuvieron lugar sobre todo en quirófano (49\%; 236 pacientes), durante procedimientos de cirugía general pediátrica (19\%; 90), de otorrinolaringología $(15 \% ; 71)$, de traumatología $(13 \% ; 61)$ y de radiología $(5 \% ; 26)$.

El tipo de IC más declarado fueron los errores de medicación $(24 \% ; 118)$, seguidos de los incidentes clínicos $(18 \%$; 87) y de los errores de equipamiento $(14 \% ; 70)$. En lo que respecta a los IC clínicos, la mayoría estuvieron relacionados con el sistema respiratorio y la vía aérea (32). Hubo 4 casos de parada cardiorrespiratoria (Figura 1).

El factor humano estuvo implicado en el $42 \%$ (79) de los IC tanto en su faceta del individuo $(25 \%$; 122$)$ como en sus relaciones en equipo $(15 \% ; 79)$, seguido de falta de cultura de seguridad $(22 \% ; 104)$, protocolos ausentes o incompletos (19\%; 102) y factores del paciente (19\%; 94). 


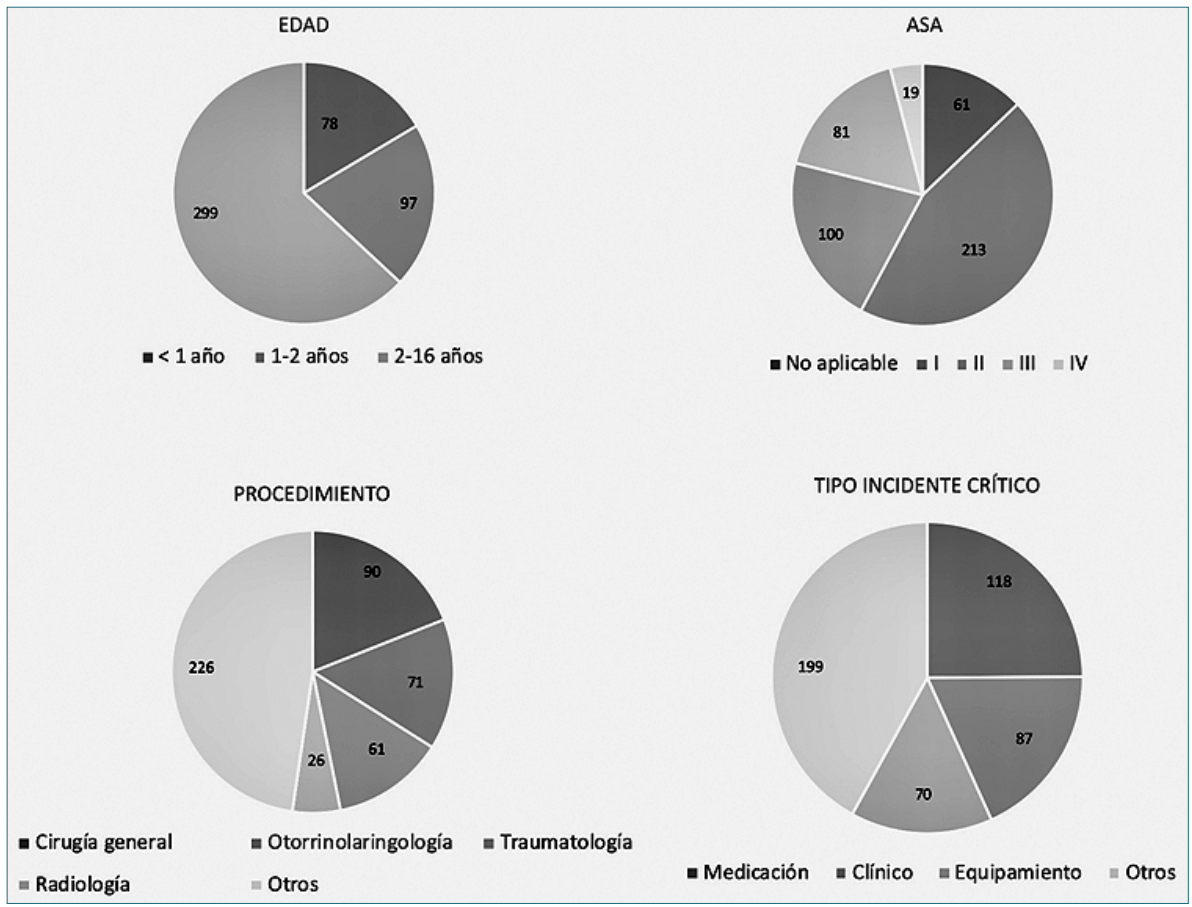

Figura 1. Distribución de los incidentes críticos declarados por edad, clasificación ASA, tipo de procedimiento y tipo de incidente, según la taxonomía utilizada. Las cifras representan el número total de incidentes declarados en cada categoría.

El 30\% (144) de los pacientes sufrieron consecuencias derivadas del incidente: el $52 \%$ (75) de ellos presentaron daño leve, el 31\% (41) daño moderado y el $14 \%$ (21) daño severo. Se registraron dos fallecimientos derivados del incidente, ambos relacionados con la vía aérea y con la dificultad para intubar en la Unidad de Cuidados Intensivos.

Se diseñaron e implementaron medidas de mejora en un $93 \%$ de los casos.

\section{Discusión}

Nuestra base de datos proporciona una visión global inicial de los IC que son declarados a nivel nacional e internacional. La tasa de declaración de ICP resulta difícilmente comparable entre la variedad de artículos disponibles, ya que numerosos autores comparan número total de anestesias pediátricas con el número de ICP declarados[6],[10],[11] y otras el número de ICP declarados con el número total de IC declarados. La realidad es que la verdadera incidencia de ICP es desconocida[5]. Nuestra impresión clínica es que la tasa española de declaración de ICP de 5\%, respecto del total comunicados traduce una franca infradeclaración. La causa es multifactorial[5], pero está estrechamente ligada al fenómeno de falta de pertenencia[12] o desvinculación con el SRIC o con la Cultura de Seguridad Moderna en general que sienten los anestesiólogos que no forman parte del Grupo de Analizadores.

Los factores de riesgo para sufrir un IC descritos en la población pediátrica incluyen la edad menor a un año, la prematuridad y la exprematuridad ${ }^{13}$. De forma análoga a otras publicaciones[6],[10],[11],[14], la mayoría de nuestros ICP se produjeron en pacientes menores de doce meses de edad. La mayoría de ellos ocurrieron en pacientes sin patología previa y tuvieron lugar en el quirófano.

Respecto al tipo de IC, los más frecuentes fueron los errores de medicación. Este tipo de IC es uno de los más declarados en la literatura[5],[15]-[17], debido fundamentalmente a tres razones: a las alertas obligatorias de farmacovigilancia, a la informatización de las prescripciones[14] y a la mayor sensibilidad sanitaria que despierta, lo que facilita su identificación, pues el concepto Error de Medicación es uno de los más extendidos en SP. En segundo lugar, destacan los IC 
clínicos, sobre todo debidos a problemas respiratorios o de vía aérea, que en muchos artículos constituyen el tipo de ICP más declarado[6],[11],[19], constituyendo una diferencia con los IC clínicos de la población adulta[19], fundamentalmente cardiovasculares o hemodinámicos.

El $70 \%$ de los IC no produjeron daño en el paciente. De los que sí lo produjeron, el $14 \%$ sufrió daño severo, comunicándose dos fallecimientos directamente derivados del incidente y relacionados con la vía aérea. Heinrich[20] ya postuló que los IC severos están a menudo precedidos por un gran número de IC que no producen daño en el paciente - near misses - o que lo hacen de forma leve; son estos IC los que proporcionan la posibilidad de implantar medidas de mejora antes de que se produzca el IC con consecuencias graves. El hecho de que los segundos IC más declarados fueran los clínicos -concretamente los respiratorios-, permite poner el foco en un dato ya publicado anteriormente, como es la existencia de brechas de seguridad en el manejo óptimo de la vía aérea en las Unidades de Cuidados Intensivos Pediátricas y Neonatales[21]. Además, tal y como describe la literatura de SP en poblaciones adultas y pediátricas[10], el factor humano fue el principal factor contribuyente implicado[22]-[24]. Nuestro sistema taxonómico no permite profundizar en el tipo de error humano ni en los mecanismos cognitivos operantes[10], lo que constituye una barrera para la implantación de medidas de mejora.

Algunas de las características de SENSAR favorecen la declaración de ICP, como el ser un sistema electrónico o dar feedback a los declarantes mediante alertas por email o sesiones de servicio. Entre sus limitaciones destaca el que no es posible averiguar el perfil concreto de cada hospital declarante - exclusivamente pediátrico, exclusivamente adulto o mixto -, ni las peculiaridades de la atención médico-quirúrgica concreta que se oferta en aquellos centros que sí realizan algún tipo de cuidado pediátrico. Recientemente, algunos hospitales terciarios de SENSAR han incorporado a sus equipos de analizadores mixtos la figura del Referente de Seguridad en Anestesia Pediátrica, esperando dar así una atención más dirigida y un mayor protagonismo a esta población tan vulnerable.

En España, SENSAR coexiste con otros SRIC, pero es el único de alcance nacional en el que todos los declarantes son anestesiólogos. Sostenemos que esto aporta una mayor precisión a la hora de declarar y otorga mayor validez a los datos obtenidos[5]. Además, la posibilidad de realizar búsquedas específicas - por ejemplo, pediátricas - permite diseñar medidas de mejora más eficientes[5] y exportar mejor los datos. De hecho, hasta donde alcanza nuestro conocimiento, este es el primer estudio que analiza ICP en España.

Entre las limitaciones de nuestro estudio se encuentran las inherentes a numerosas bases online de comunicación - que conducen a errores en la clasificación de IC[5],[14] -, la infradeclaración[10] y las debidas al análisis retrospectivo de la información limitada que contiene una declaración escrita.

Es necesario diseñar estudios que arrojen luz sobre las causas de la ineficiencia relativa de los SRIC y las razones de que no hayan alcanzado el potencial esperado[25] y determinar los factores que condicionan una menor tasa de comunicación de IC en el ámbito pediátrico con respecto al adulto.

\section{Referencias}

1. Smith AF, Mahajan RP. National critical incident reporting: improving patient safety. Br J Anaesth. 2009 Nov;103(5):623-5. https:// doi.org/10.1093/bja/aep273 PMID:19837805

2. Spigelman AD, Swan J. Incident reporting: patient and clinician safety. ANZ J Surg. 2005;75(8):657-61. https:// doi.org/10.1111/j.14452197.2005.03482.x PMID:16076327
3. van Beuzekom M, Boer F, Akerboom S, Hudson P. Patient safety: latent risk factors. $\mathrm{Br} \mathrm{J}$ Anaesth. 2010 Jul;105(1):529. https://doi.org/10.1093/bja/ aeq135 PMID:20551026

4. Bunchungmongkol N, Somboonviboon W, Suraseranivongse $S$, Vasinanukorn $M$, Chau-in W, Hintong T. Pediatric anesthesia adverse events: the Thai Anesthesia Incidents Study (THAI Study) database of 25,098 cases. J Med Assoc Thai. 2007 Oct;90(10):2072-9.
PMID:18041426

5. MacLennan Al, Smith AF. An analysis of critical incidents relevant to pediatric anesthesia reported to the UK National Reporting and Learning System, 2006-2008. Paediatr Anaesth. 2011 Aug;21(8):8417. https://doi.org/10.1111/ j.1460-9592.2010.03421.x PMID:21054660

6. Tay CL, Tan GM, Ng SB. Critical incidents in paediatric anaesthesia: an audit of 10000 anaesthetics in Singapore. Paediatr 
Anaesth. $2001 \mathrm{Nov} ; 11(6): 711-$

8. https://doi.org/10.1046/

j.1460-9592.2001.00767.x

PMID:11696149

7. Abbasi S, Khan FA, Khan S. Pediatric critical incidents reported over 15 years at a tertiary care teaching hospital of a developing country. J Anaesthesiol Clin Pharmacol. 2018 Jan-Mar;34(1):78-83. PMID:29643628

8. Beckmann U, Baldwin I, Hart GK, Runciman WB. The Australian Incident Monitoring Study in Intensive Care: AIMS-ICU. An analysis of the first year of reporting. Anaesth Intensive Care. 1996 Jun;24(3):320-9. https://doi.g/1 $0.1177 / 0310057 \times 9602400304$ PMID:8805886

9. Vincent $C$, Taylor-Adams S, Stanhope N. Framework for analysing risk and safety in clinical medicine. BMJ. 1998 Apr;316(7138):11547. https://doi.org/10.1136/ bmj.316.7138.1154 PMID:9552960

10. Marcus R. Human factors in pediatric anesthesia incidents. Paediatr Anaesth. 2006 Mar;16(3):242-50. https://doi.org/10.1111/ j.1460-9592.2005.01771.x PMID:16490087

11. Murat I, Constant I, Maud'huy $H$. Perioperative anaesthetic morbidity in children: a database of 24,165 anaesthetics over a 30-month period. Paediatr Anaesth. 2004 Feb;14(2):15866. https://doi.org/10.1111/ j.1460-9592.2004.01167.x PMID:14962332

12. Sharma S, Smith AF, Rooksby J, Gerry B. Involving users in the design of a system for sharing lessons from adverse incidents in anaesthesia. Anaesthesia. 2006 Apr;61(4):350-4. https://doi.org/10.1111/

j.1365-2044.2006.04542.x PMID:16548954

13. Wan S, Siow YN, Lee SM, Ng A. Audits and critical incident reporting in paediatric anaesthesia: lessons from 75,331 anaesthetics. Singapore Med J. 2013 Feb;54(2):69-74. https://doi. org/10.11622/smedj.2013027 PMID:23462829

14. Días R, Dave N, Chiluveru S, Garasia M. Critical incidents in paediatric anaesthesia: A prospective analysis over a 1 year period. Indian J Anaesth. 2016 Nov;60(11):801-6. https://doi. org/10.4103/0019-5049.193658 PMID:27942052

15. Bordun LA, Butt W. Drug errors in intensive care. J Paediatr Child Health. 1992 Aug;28(4):309-11. https://doi. org/10.1111/j.1440-1754.1992. tb02674.x PMID:1497959

16. Wilson DG, McArtney RG, Newcombe RG, McArtney RJ, Gracie J, Kirk CR, et al. Medication errors in paediatric practice: insights from a continuous quality improvement approach. Eur J Pediatr. 1998 Sep;157(9):76974. https://doi.org/10.1007/ s004310050932 PMID:9776539

17. Raju TN, Kecskes S, Thornton JP, Perry M, Feldman S. Medication errors in neonatal and paediatric intensive-care units. Lancet. 1989 Aug;2(8659):374-6. https://doi.org/10.1016/S01406736(89)90548-5 PMID:2569561

18. Aronson JK. Medication errors: definitions and classification. Br J Clin Pharmacol. 2009 Jun;67(6):599-604. https://doi.org/10.1111/ j.1365-2125.2009.03415.x PMID:19594526

19. Van der Walt JH, Sweeney DB, Runciman WB, Webb RK. The Australian Incident Monitoring
Study. Paediatric incidents in anaesthesia: an analysis of 2000 incident reports. Anaesth Intensive Care. 1993 Oct;21(5):655-8. https://doi.g/10.1177/031005 7X9302100529 PMID:8273893

20. Yorio PL, Moore SM. Examining Factors that Influence the Existence of Heinrich's Safety Triangle Using Site-Specific H\&S Data from More than 25,000 Establishments. Risk Anal. 2018 Apr;38(4):839-52. https:// doi.org/10.1111/risa.12869 PMID:28768045

21. Foy KE, Mew E, Cook TM, Bower J, Knight P, Dean S, et al. Paediatric intensive care and neonatal intensive care airway management in the United Kingdom: the PIC-NIC survey. Anaesthesia. 2018 Nov;73(11):133744. https://doi.org/10.1111/ anae.14359 PMID:30112809

22. Reason J. Safety in the operating theatre. Part 2: human error and organizational failure. Curr Anaesth Crit Care. 1995;6(2):121-6. https:// doi.org/10.1016/S09537112(05)80010-9.

23. Reason J. Human Error. Cambridge: Cambridge University Press, 1990: 53-96, 173-216. https://doi.org/10.1017/ CBO9781139062367.

24. Phipps D, Meakin GH, Beatty PC, Nsoedo C, Parker D. Human factors in anaesthetic practice: insights from a task analysis. $\mathrm{Br} J$ Anaesth. 2008 Mar;100(3):33343. https://doi.org/10.1093/bja/ aem392 PMID:18238839

25. Stavropoulou C, Doherty C, Tosey $P$. How effective are incidentreporting systems for improving patient safety? A systematic literature review. Milbank Q. 2015 Dec;93(4):826-66. https://doi. org/10.1111/1468-0009.12166 PMID:26626987 